\title{
1-[1-(4-Chlorobenzenesulfonyl)-1H-indole-3-yl]-3- [4-(pyridin-2-yl)piperazin-1-yl]propan-1-one
}

\author{
Benjamín Diethelm, Sebastián Almendras (iD) and Gonzalo Recabarren-Gajardo * (iD) \\ Laboratorio de Síntesis de Heterociclos Bioactivos, Departamento de Farmacia, Facultad de Química, \\ Pontificia Universidad Católica de Chile, Casilla 306, Avda. Vicuña Mackenna 4860, Macul 7820436, \\ Santiago, Chile; bmdiethelm@uc.cl (B.D.); sialmend@uc.cl (S.A.) \\ * Correspondence: grecabarren@uc.cl; Tel.: +56-223-541-418
}

Received: 17 March 2018; Accepted: 16 April 2018; Published: 18 April 2018

Abstract: The title compound was prepared by an aza-Michael addition reaction between 1-[1-(4chlorobenzenesulfonyl)-1H-indole-3-yl]prop-2-en-1-one and 2-piridylpiperazine catalyzed by $\mathrm{SiO}_{2}$. The structural identity of the title compound was proven by elemental analysis and spectroscopic methods (IR, NMR). The compound was assayed in a binding assay at the 5- $\mathrm{HT}_{6}$ receptor, showing poor affinity.

Keywords: aza-Michael addition; indole; serotonin; 5- $\mathrm{HT}_{6}$; arylpiperazine; arylsulfonylindole

\section{Introduction}

The serotonin receptor subtype $6\left(5-\mathrm{HT}_{6}\right)$, a metabotropic receptor located exclusively within the central nervous system [1-3], is considered a promising target for the treatment of several illnesses such as Alzheimer's disease, obesity, and major depressive disorder [4-6]. A 5- $\mathrm{HT}_{6}$ antagonist pharmacophore has been previously described in the literature [7]. In our efforts to prepare potent and highly selective antagonists targeting $5-\mathrm{HT}_{6}$ based on this reported pharmacophore [8,9], we performed the synthesis of the title compound 1-[1-(4-chlorobenzenesulfonyl)$1 H$-indole-3-yl]-3-[4-(pyridin-2-yl)piperazin-1-yl]propan-1-one and evaluated its affinity for the 5- $\mathrm{HT}_{6}$ receptor in a standard binding assay.

\section{Results and Discussion}

Title compound 5 was synthesized through a series of reactions beginning from commercial indole, and using methods reported in the literature (Scheme 1). First, indole was formylated in C-3 employing the Vilsmeier-Haack synthesis, affording formylindole $\mathbf{1}$ in excellent yield [10]. Afterwards, $\mathrm{N}$-sulfonylation in basic media gave $\mathrm{N}$-(4-chlorobenzenesulfonyl)-3-formylindole 2 in good yield [8]. A nucleophilic attack on the formyl group with the commercially available Grignard reagent vinylmagnesium bromide led to secondary allylic alcohol 3, which was oxidized to the corresponding $\alpha, \beta$-unsaturated ketone 4 employing $\mathrm{MnO}_{2} / \mathrm{MgSO}_{4}$ [10]. Synthesis of the title compound involved an aza-Michael addition reaction, with 2-pyridylpiperazine. This reaction was initially attempted using microwave irradiation [10]; however, yields were poor, and degradation products were observed. We therefore attempted a reflux reaction in acetonitrile, employing $\mathrm{SiO}_{2}$ as a catalyst [11]. These conditions afforded the desired product 5, which was obtained as a crude product and then purified by gravity column chromatography in $89 \%$ yield. Spectroscopic data confirmed the structure of the product.

In the context of our interest to produce highly active antagonists towards the $5-\mathrm{HT}_{6}$ receptor, 5 was tested in a standard radioligand competition binding assay, using membranes of HEK-293 cells expressing a recombinant human $5-\mathrm{HT}_{6}$ receptor, as previously described $[8,12]$. The product 
was assayed as a free base at eight concentrations, in triplicate, to obtain the dose-response curve, determine the $\mathrm{IC}_{50}$ value and calculate the $\mathrm{Ki}$ through the Cheng-Prusoff equation [13]. Using this approach, it was determined that the $\mathrm{Ki}$ was $1.33 \mu \mathrm{M}$, and that the $\mathrm{IC}_{50}$ had a magnitude of $1.38 \mu \mathrm{M}$, which is regarded as a poor affinity for the receptor (for the dose-response curve, see Figure S16).

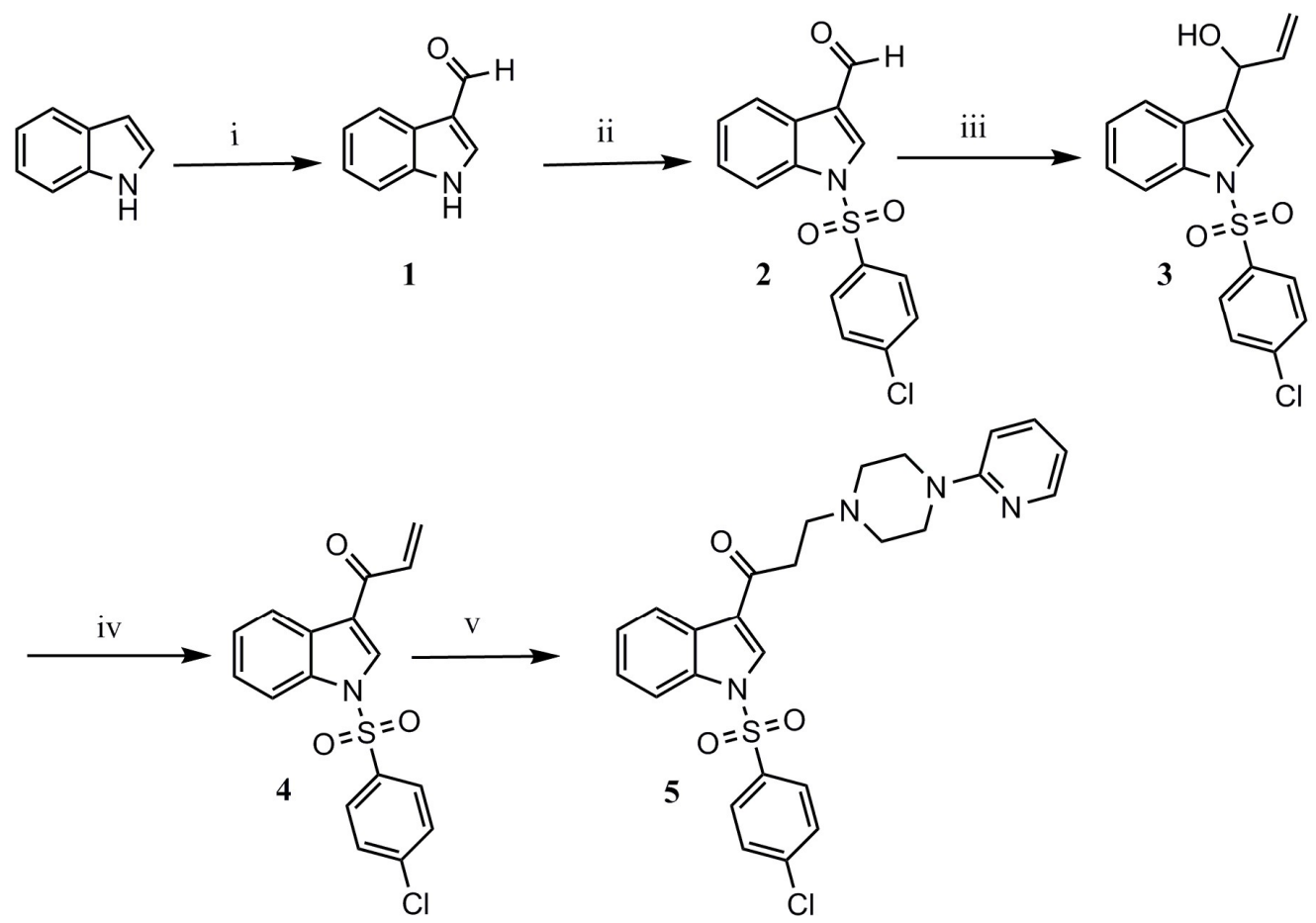

Scheme 1. Synthesis of 1-[1-(4-chlorobenzenesulfonyl)-1H-indol-3-yl]-3-[4-(pyridin-2-yl)piperazin-1yl]propan-1-one. Reagents and conditions: (i) $\mathrm{POCl}_{3}, \mathrm{DMF},{ }^{\circ} \mathrm{C}, 30 \mathrm{~min}$; (ii) $\mathrm{CH}_{2} \mathrm{Cl}_{2}, \mathrm{Et}_{3} \mathrm{~N}, \mathrm{DMAP}$, 4-chlorobenzenesulfonyl chloride; (iii) vinylmagnesium bromide, anhydrous THF, r.t., 8-10 h; (iv) anhydrous $\mathrm{CH}_{2} \mathrm{Cl}_{2}, \mathrm{MnO}_{2}$, anhydrous $\mathrm{MgSO}_{4} ;$ (v) $\mathrm{SiO}_{2}, \mathrm{CH}_{3} \mathrm{CN}$, 2-pyridylepiperazine, reflux, 12-20 h.

\section{Materials and Methods}

\subsection{Materials}

Reagents were purchased from commercial suppliers, specifically Merck (Darmstadt, Germany) and Sigma-Aldrich (St. Louis, MO, USA), and used without further purification. Solvents were purchased from commercial suppliers and were purified by distillation prior to their use.

\subsection{Instrumentation}

Melting points were determined on a Stuart Scientific SMP30 apparatus (Bibby Scientific Limited, Stone, UK), employing open-glass capillaries. Infrared spectra were recorded on a BRUKER Vector 22 spectrometer using $\mathrm{KBr}$ pellets. NMR spectra were recorded on a Bruker Avance III HD 400 (Billerica, MA, USA) at $400 \mathrm{MHz}$ for ${ }^{1} \mathrm{H}$ and $100 \mathrm{MHz}$ for ${ }^{13} \mathrm{C}-\mathrm{NMR}$ spectra were recorded in $\mathrm{CDCl}_{3}$, using the solvent signal as a reference. The chemical shifts are expressed in ppm ( $\delta$ scale) downfield from tetramethylsilane (TMS) and coupling constants values $(J)$ are given in Hertz. The following multiplicity abbreviations were utilized: singlet (s), broad signal (bs); doublet (d); doublet of doublets (dd); multiplet (m). Elemental analyses were performed on a FISONS EA 1108 CHNS-O elemental analyzer (Thermo Scientific, Waltham, MA, USA). Radioligand binding studies were performed by Scottish Biomedical Drug Discovery. 


\subsection{Synthesis}

3.3.1. 3-Formylindole (1)<smiles>O=Cc1c[nH]c2ccccc12</smiles>

$\mathrm{POCl}_{3}(0.8 \mathrm{~mL}, 1.34 \mathrm{~g}, 8.74 \mathrm{mmol})$ was added dropwise over DMF (5 mL, $\left.4.72 \mathrm{~g}, 64.58 \mathrm{mmol}\right)$ in a round-bottomed flask at $0{ }^{\circ} \mathrm{C}$. Afterwards, indole $(1 \mathrm{~g}, 8.54 \mathrm{mmol})$ was dissolved in another round-bottomed flask containing DMF $(5 \mathrm{~mL})$. The $\mathrm{POCl}_{3}$ solution was slowly added to the indole solution. The mixture was stirred at $0{ }^{\circ} \mathrm{C}$ for $30 \mathrm{~min}$, after which the contents were poured over a water-ice mixture and the $\mathrm{pH}$ was regulated to 12. A pale yellow precipitate was formed, which was filtered and dried in a stove. This product was used in subsequent syntheses without further purification. Yield: 95\%. m.p.: $189.1-192.5{ }^{\circ} \mathrm{C}$ (lit.: $193-195{ }^{\circ} \mathrm{C}$ [14]). ${ }^{1} \mathrm{H}-\mathrm{NMR}$ (400 MHz, DMSO- $\left.d_{6}\right)$ : $\delta$ (ppm) 12.18 (s, 1H; NH); 9.99 (s, 1H; CHO); 8.31 (s, 1H; H1); 8.17 (d, J = 7.2 Hz; 1H; H2); 7.56 (d, $J=7.6 \mathrm{~Hz} ; 1 \mathrm{H} ; \mathrm{H} 5) ; 7.23-7.31$ (m, 2H; H3, H4). ${ }^{13} \mathrm{C}-\mathrm{NMR}\left(101 \mathrm{MHz}, \mathrm{DMSO}-d_{6}\right): \delta 185.43 ; 138.86 ; 137.55$; 124.63; 123.93; 122.60; 121.32; 118.68; 112.90. IR (cm $\left.{ }^{-1}\right): 3169(\mathrm{NH}) ; 2932\left(\mathrm{CH}_{\mathrm{Ar}}\right) ; 1634$ (CHO). Anal. calculated for $\mathrm{C}_{9} \mathrm{H}_{7} \mathrm{NO}(\%)$ : C: 74.47; $\mathrm{H}: 4.86 ; \mathrm{N}: 9.65$. Anal. found for $\mathrm{C}_{9} \mathrm{H}_{7} \mathrm{NO}(\%)$ : C: 74.02; $\mathrm{H}: 4.79$; $\mathrm{N}: 9.92$.

3.3.2. 1-(4-Chlorobenzenesulfonyl)-3-formylindole (2)<smiles>[CH2+]c1ccc(S(=O)(=O)n2cc(C=O)c3ccccc32)cc1</smiles>

2

Triethylamine $\left(\mathrm{Et}_{3} \mathrm{~N} ; 0.44 \mathrm{~mL}, 0.318 \mathrm{~g}, 3.14 \mathrm{mmol}\right)$ and 4-dimethylaminopyridine (DMAP; $0.048 \mathrm{~g}$, $0.31 \mathrm{mmol})$ were added over a stirred solution of $\mathbf{1}(0.46 \mathrm{~g}, 3.14 \mathrm{mmol})$ in $\mathrm{CH}_{2} \mathrm{Cl}_{2}(30 \mathrm{~mL})$. After $10 \mathrm{~min}$, $p$-chlorobenzenesulfonyl chloride $(0.796 \mathrm{~g}, 3.77 \mathrm{mmol})$ is added. Reaction progress was monitored by TLC until the starting materials had disappeared. At this point, the reaction was quenched by adding a $1 \mathrm{M} \mathrm{HCl}$ solution. The organic layer was extracted with EtOAc $(3 \times 20 \mathrm{~mL})$ and dried over anhydrous $\mathrm{Na}_{2} \mathrm{SO}_{4}$. Solvent was removed under a vacuum, affording a crude product that was purified by gravity column chromatography, employing silica gel as adsorbent and $\mathrm{CH}_{2} \mathrm{Cl}_{2}$ as eluent. This purification affords a white crystalline solid. Yield: $76 \%$. m.p.: $152.8-155.1{ }^{\circ} \mathrm{C}$ (lit.: $152-154{ }^{\circ} \mathrm{C}$ [15]). ${ }^{1} \mathrm{H}-\mathrm{NMR}$ (400 MHz, CDCl $): \delta 10.09$ (s, 1H; CHO); 8.26 (d, J = $7.3 \mathrm{~Hz} ; 1 \mathrm{H} ; \mathrm{H} 2) ; 8.21$ (s, 1H; H1); 7.92 (d, J = 8.2 Hz; 1H; H5); 7.89 (d, J = 8.7 Hz; 2H; H1', H2'); 7.45 (d, J = 8.7 Hz; 2H; H3', H4'); 7.35-7.42 (m, 2H; H3, H4). ${ }^{13} \mathrm{C}-\mathrm{NMR}\left(101 \mathrm{MHz}, \mathrm{CDCl}_{3}\right): \delta 185.69 ; 142.06 ; 136.34 ; 136.09 ; 135.54 ; 130.50 ; 128.96 ; 126.98 ; 126.76$; 125.75; 123.20; 123.18; 113.52. IR ( $\left.\mathrm{cm}^{-1}\right)$ : $3137\left(\mathrm{CH}_{\text {het }}\right) ; 3086\left(\mathrm{CH}_{\mathrm{ar}}\right) ; 1664(\mathrm{C}=\mathrm{O}) ; 1380\left(\mathrm{SO}_{2}\right) ; 1182$ $\left(\mathrm{SO}_{2}\right)$. Anal. calculated for $\mathrm{C}_{15} \mathrm{H}_{10} \mathrm{ClNO}_{3} \mathrm{~S}(\%)$ : C: 56.34; $\mathrm{H}: 3.15 ; \mathrm{N}: 4.38 ; \mathrm{S}: 10.03$ Anal. found for $\mathrm{C}_{15} \mathrm{H}_{10} \mathrm{ClNO}_{3} \mathrm{~S}$ (\%): C: 56.38; H: 3.20; N: 4.94; S: 10.16. 
3.3.3. 1-[1-(4-Chlorobenzenesulfonyl)-1H-indole-3-yl]-prop-2-en-1-ol (3)<smiles></smiles>

3

2 was added to a round-bottomed flask, which was purged with $\mathrm{N}_{2}$. Afterwards, anhydrous THF and vinylmagnesium bromide were added. The contents were stirred for 8-10 h, after which the reaction was quenched adding a saturated $\mathrm{NH}_{4} \mathrm{Cl}$ solution. The product was extracted with $\mathrm{CH}_{2} \mathrm{Cl}_{2}$ $(3 \times 20 \mathrm{~mL})$ and dried over $\mathrm{Na}_{2} \mathrm{SO}_{4}$. Solvent was removed under vacuum, affording an orange-colored oil, which was purified by gravity column chromatography, employing silica gel as adsorbent and $\mathrm{CH}_{2} \mathrm{Cl}_{2}$ as eluent. The purified product corresponds to a yellow oil. Yield: $83 \%$. ${ }^{1} \mathrm{H}-\mathrm{NMR}(400 \mathrm{MHz}$, $\left.\mathrm{CDCl}_{3}\right): \delta 7.94(\mathrm{~d}, J=8.3 \mathrm{~Hz} ; 1 \mathrm{H} ; \mathrm{H} 2) ; 7.79\left(\mathrm{~d}, J=8,6 \mathrm{~Hz} ; 2 \mathrm{H} ; \mathrm{H} 1^{\prime}, \mathrm{H} 2^{\prime}\right) ; 7.62$ (d, J = $\left.7.8 \mathrm{~Hz} ; 1 \mathrm{H} ; \mathrm{H} 5\right)$; 7.50 (s, 1H; H1); 7.30-7.36 (m, 3H; H3, H3' , H4'); 7.23 (t, J = 7.5 Hz; 1H; H4); 6.08-6.12 (m, 1H; H1"); 5.40-5.44 (m, 2H; H3", H4"); 5.27 (d, J $\left.J_{\text {cis }}=10.2 \mathrm{~Hz} ; 1 \mathrm{H} ; \mathrm{H} 2 "\right) ; 2.25$ (bs, $\left.1 \mathrm{H} ; \mathrm{OH}\right) .{ }^{13} \mathrm{C}-\mathrm{NMR}(101 \mathrm{MHz}$, $\left.\mathrm{CDCl}_{3}\right): \delta 140.97 ; 138.89 ; 136.82 ; 135.87 ; 130.04 ; 129.39 ; 128.61 ; 125.58 ; 125.26 ; 123.97 ; 123.45 ; 121.19$;

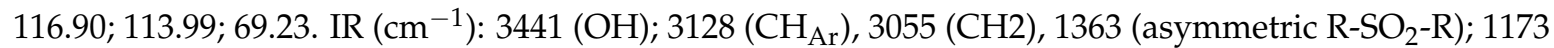
(symmetric $\mathrm{R}-\mathrm{SO}_{2}-\mathrm{R}$ ). Anal. calculated for $\mathrm{C}_{21} \mathrm{H}_{17} \mathrm{NO}_{3} \mathrm{~S}$ (\%): C: 69.40; $\mathrm{H}: 4.71 ; \mathrm{N}: 3.85 ; \mathrm{S}, 8.82$. Anal. found for $\mathrm{C}_{21} \mathrm{H}_{17} \mathrm{NO}_{3} \mathrm{~S}$ (\%): C: 69.22; $\mathrm{H}: 5.07 ; \mathrm{N}: 3.56 ; \mathrm{S}: 8.93$.

3.3.4. 1-[1-(4-Chlorobenzenesulfonyl)-1H-indole-3-yl]-prop-2-en-1-one (4)<smiles></smiles>

A solution of $3(0.382 \mathrm{~g}, 1.10 \mathrm{mmol})$ in anhydrous $\mathrm{CH}_{2} \mathrm{Cl}_{2}(30 \mathrm{~mL})$ was prepared. Afterwards, $\gamma-\mathrm{MnO}_{2}(1.91 \mathrm{~g})$ and anhydrous $\mathrm{MgSO}_{4}(0.127 \mathrm{~g}, 1.10 \mathrm{mmol})$ were added. The mixture was stirred vigorously while monitoring the reaction progress by TLC. Once oxidation was complete, $\mathrm{MnO}_{2}$ was removed by filtration, and the solvent was removed under a vacuum. The resulting crude product was purified by gravity column chromatography, employing silica gel as adsorbent and $\mathrm{CH}_{2} \mathrm{Cl}_{2}$ as eluent, affording a pale yellow amorphous solid. Yield: $51 \%$. m.p.: $137.7-140.1{ }^{\circ} \mathrm{C} .{ }^{1} \mathrm{H}-\mathrm{NMR}(400 \mathrm{MHz}$, $\left.\mathrm{CDCl}_{3}\right): \delta 8.42(\mathrm{~d}, J=6.7 \mathrm{~Hz} ; 1 \mathrm{H} ; \mathrm{H} 2) ; 8.26(\mathrm{~s}, 1 \mathrm{H} ; \mathrm{H} 1) ; 7.94(\mathrm{~d}, J=7.3 \mathrm{~Hz}, 1 \mathrm{H} ; \mathrm{H} 5) ; 7.88(\mathrm{~d}, J=8.4 \mathrm{~Hz}$; $\left.2 \mathrm{H} ; \mathrm{H}^{\prime}, \mathrm{H} 2^{\prime}\right) ; 7.45$ (d, J = 8.5 Hz; 2H; H3' $\left.{ }^{\prime} \mathrm{H}^{\prime}{ }^{\prime}\right), 7.38-7.42$ (m, 2H; H3, H4); 7.07 (dd, J Jrans $=17.0$ and $\left.J_{\text {cis }}=10,5 \mathrm{~Hz} ; 1 \mathrm{H} ; \mathrm{H} 1 "\right) ; 6.51\left(\mathrm{~d}, J_{\text {trans }}=17.0 \mathrm{~Hz}, 1 \mathrm{H} ; \mathrm{H} 3{ }^{\prime \prime}\right) ; 5.90\left(\mathrm{~d}, J_{\mathrm{cis}}=10.5 \mathrm{~Hz} ; 1 \mathrm{H} ; \mathrm{H} 2^{\prime \prime}\right) .{ }^{13} \mathrm{C}-\mathrm{NMR}$ 
$\left(101 \mathrm{MHz}, \mathrm{CDCl}_{3}\right): \delta 185.77 ; 141.89 ; 136.25 ; 135.33 ; 133.43 ; 132.20 ; 130.42 ; 129.22 ; 128.88 ; 128.41 ; 126.64 ;$ 125.57; 123.88; 122.27; 113.37. IR ( $\left.\mathrm{cm}^{-1}\right): 3122-3122\left(\mathrm{CH}_{\mathrm{Ar}}\right), 3054\left(=\mathrm{CH}_{2}\right), 1665(\mathrm{C}=\mathrm{O}), 1603(\mathrm{C}=\mathrm{C}), 1373$ (asymmetric $\mathrm{R}-\mathrm{SO}_{2}-\mathrm{R}$ ); 1174 (symmetric $\mathrm{R}-\mathrm{SO}_{2}-\mathrm{R}$ ). Anal. calculated for $\mathrm{C}_{17} \mathrm{H}_{12} \mathrm{ClNO}_{3} \mathrm{~S}$ (\%): C: 59.05; $\mathrm{H}: 3.50 ; \mathrm{N}: 4.05$; S: 9.27. Anal. found for $\mathrm{C}_{17} \mathrm{H}_{12} \mathrm{ClNO}_{3} \mathrm{~S}(\%)$ : C: 58.89; $\mathrm{H}: 3.67 ; \mathrm{N}: 4.59 ;$ S: 9.60.

3.3.5. 1-[1-(4-Chlorobenzenesulfonyl)-1H-indole-3-yl]-3-[4-(pyridin-2-yl)piperazin-1-yl]propan-1-one (5)

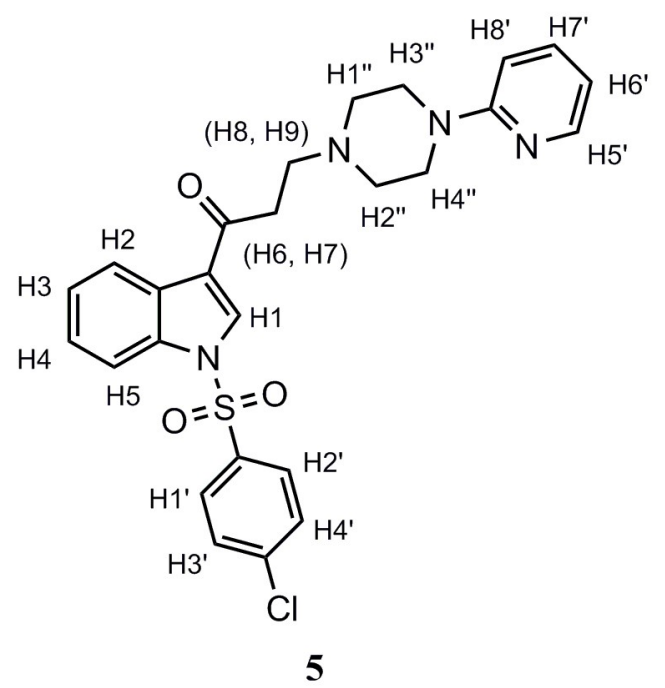

$4(0.185 \mathrm{~g}, 0.53 \mathrm{mmol}), 2$-pyridylpiperazine $(0.105 \mathrm{~g}, 0.64 \mathrm{mmol})$ and a catalytic amount of silica gel $\left(\mathrm{SiO}_{2}, 0.01-0.1 \mathrm{~g}\right)$, were dissolved in $\mathrm{CH}_{3} \mathrm{CN}(40 \mathrm{~mL})$. The mixture was refluxed for 12 to $20 \mathrm{~h}$, and the reaction progress was monitored by TLC. The product, once purified by gravity column chromatography employing silica gel as adsorbent and EtOAc as eluent, corresponds to an amorphous white solid. Yield: 89\%. m.p.: $154.9-158.9{ }^{\circ} \mathrm{C} ;{ }^{1} \mathrm{H}-\mathrm{NMR}\left(400 \mathrm{MHz}, \mathrm{CDCl}_{3}\right): \delta 8.34(\mathrm{~d}, \mathrm{~J}=7.3 \mathrm{~Hz} ; 1 \mathrm{H}$; H2); 8.26 (s, 1H; H1); 8.20 (bs, 1H; H5'); 7.92 (d, J = 7.5 Hz; 1H; H5); 7.87 (d, J = 8.1 Hz; 2H; H1', H2'); 7.46 (m; 3H; H3', H4', H7'); 7.38 (m; 2H; H3, H4); 6.65 (m; 2H; H6', H8'); 3.56 (bs, 4H; H3" , H4"); 3.15 (t, $J=7.0 \mathrm{~Hz}, 2 \mathrm{H} ; \mathrm{H6}, \mathrm{H7}) ; 2.91$ (t, $J=7.0 \mathrm{~Hz} ; 2 \mathrm{H} ; \mathrm{H8}, \mathrm{H} 9) ; 2.64$ (bs, 4H; H1", H2"). ${ }^{13} \mathrm{C}-\mathrm{NMR}(101 \mathrm{MHz}$, $\left.\mathrm{CDCl}_{3}\right): \delta 195.01 ; 159.88 ; 148.38 ; 141.89 ; 137.86 ; 136.29 ; 135.27 ; 131.99 ; 130.43 ; 128.85 ; 128.05 ; 126.46 ;$ $125.55 ; 123.71 ; 122.19 ; 113.79 ; 113.36 ; 107.49 ; 53.59 ; 53.50 ; 45.61 ; 38.31 . \mathrm{IR}\left(\mathrm{cm}^{-1}\right): 3141-3065\left(\mathrm{CH}_{\mathrm{Ar}}\right)$, $1664(\mathrm{C}=\mathrm{O}), 1382\left(\mathrm{R}-\mathrm{SO}_{2}-\mathrm{R}\right), 1174\left(\mathrm{R}-\mathrm{SO}_{2}-\mathrm{R}\right)$. Anal. calculated for $\mathrm{C}_{26} \mathrm{H}_{25} \mathrm{ClN}_{4} \mathrm{O}_{3} \mathrm{~S}(\%)$ : C: 61.35; $\mathrm{H}$ : 4.95; N: 11.01; S: 6.30. Anal. found for $\mathrm{C}_{26} \mathrm{H}_{25} \mathrm{ClN}_{4} \mathrm{O}_{3} \mathrm{~S}$ (\%): C: 61.83; H: 5.04; N: 10.56; S: 6.05.

\subsection{Radioligand Binding Studies}

Affinity of the title compound for 5- $\mathrm{HT}_{6}$ receptors was evaluated using HEK-293 cells expressing human $5-\mathrm{HT}_{6} \mathrm{R}$, employing the iodinated specific radioligand $\left[{ }^{125} \mathrm{I}\right]-\mathrm{SB}-258585$ (4-iodo-N-[4-methoxy-3-(4-methyl-piperazin-1-yl)-phenyl]-benzenesulfonamide); $\mathrm{Kd}=1.3 \mathrm{nM}$; $2200 \mathrm{Ci} / \mathrm{mmol})$. Competitive inhibition assays were performed according to standard procedures, briefly detailed below.

Fractions of $45 \mu \mathrm{L}$ of diluted $5-\mathrm{HT}_{6}$ membrane preparation were incubated at $27^{\circ} \mathrm{C}$ for $180 \mathrm{~min}$ with $255-\mathrm{HT}_{6} \mathrm{R}$ L of $\left[{ }^{125} \mathrm{I}\right]-\mathrm{SB}-258585(0.2 \mathrm{nM})$ and $255-\mathrm{HT}_{6} \mathrm{R} \mathrm{L}$ of WGA PVT SPA beads $(4 \mathrm{mg} / \mathrm{mL})$, in the presence of increasing concentrations $\left(10^{-11}\right.$ to $\left.10^{-4} \mathrm{M}\right)$ of the competing drug $(5 \mu \mathrm{L})$ or DMSO, in a final volume of $100 \mu \mathrm{L}$ of assay buffer (50 mM Tris, $120 \mathrm{mM} \mathrm{NaCl}$, pH 7.4). Non-specific binding was determined by radioligand binding in the presence of a saturating concentration of $100 \mu \mathrm{M}$ of clozapine. Binding of $\left.{ }^{125} \mathrm{I}\right]-\mathrm{SB}-258585$ to $5-\mathrm{HT}_{6}$ receptors directly correlates to an increase in signal that was read on a Perkin Elmer Topcount NXT HTS (PerkinElmer, Waltham, MA, USA). Compounds were tested at eight concentrations, in triplicate. Clozapine was used as an internal standard for comparison. Data generated were analyzed using GraphPad Prism version 7.0 (GraphPad Software Inc., La Jolla, CA, USA). A linear regression line of data points was plotted, from which the 
concentration of the competing ligand that displaces $50 \%$ of the specific binding of the radioligand $\left(\mathrm{IC}_{50}\right.$ value) was determined and the Ki value was calculated based upon the Cheng-Prusoff equation, $\mathrm{Ki}=\mathrm{IC}_{50} /(1+\mathrm{L} / \mathrm{Kd})$, where $\mathrm{L}$ is the concentration of free radioligand used in the assay and $\mathrm{Kd}$ is the dissociation constant of the radioligand for the receptor.

\section{Conclusions}

In this work, we report the synthesis of 1-[1-(4-chlorobenzenesulfonyl)-1H-indole-3-yl]-3[4-(pyridin-2-yl)piperazin-1-yl]propan-1-one by a route starting from commercial indole. The final step of this route, which leads to the title compound, involved an aza-Michael addition reaction between 1-[1-(4-chlorobenzenesulfonyl)- $1 \mathrm{H}$-indole-3-yl]prop-2-en-1-one and 2-piridylpiperazine. Use of $\mathrm{SiO}_{2}$ as a catalyst afforded the desired final product in excellent yield. Both the title compound and its precursors had their structural identity proven employing spectroscopic methods, and a complete physical characterization was provided for all of them. In the context of our efforts to produce potent and selective novel $5-\mathrm{HT}_{6}$ receptor antagonists, we measured the title compound's affinity for this receptor employing a standard radioligand binding assay. Unfortunately, the results of said assay revealed that the title compound displays a poor affinity for the receptor. Nevertheless, this study highlights an interesting synthetic route to the preparation of arylsulfonylindolepropanones, thus providing orientation for the design of future ligands acting as 5- $\mathrm{HT}_{6}$ receptor antagonists.

Supplementary Materials: The following are available online, Figure S1: ${ }^{1} \mathrm{H}-\mathrm{NMR}$ spectrum of 1; Figure S2: ${ }^{13} \mathrm{C}-\mathrm{NMR}$ spectrum of 1; Figure S3: ${ }^{1} \mathrm{H}-\mathrm{NMR}$ spectrum of 2; Figure S4: ${ }^{13} \mathrm{C}-\mathrm{NMR}$ spectrum of 2; Figure S5: ${ }^{1} \mathrm{H}-\mathrm{NMR}$ spectrum of 3; Figure S6: ${ }^{13} \mathrm{C}-\mathrm{NMR}$ spectrum of 3; Figure S7: ${ }^{1} \mathrm{H}-\mathrm{NMR}$ spectrum of 4; Figure S8: ${ }^{13} \mathrm{C}-\mathrm{NMR}$ spectrum of 4; Figure S9: ${ }^{1} \mathrm{H}-\mathrm{NMR}$ spectrum of 5; Figure S10: ${ }^{13} \mathrm{C}-\mathrm{NMR}$ spectrum of 5; Figure S11: IR spectrum of 1; Figure S12: IR spectrum of 2; Figure S13: IR spectrum of 3; Figure S14: IR spectrum of 4; Figure S15: IR spectrum of 5; Figure S16: Dose-response curve for the 5- $\mathrm{HT}_{6}$ receptor of 5.

Acknowledgments: This work was supported by FONDECYT No. 11121418 project.

Author Contributions: B.D.: Literature research, writing of manuscript; G.R.-G.: Synthesis planning, IR and NMR interpretation, proofreading of manuscript; S.A.: Experimental synthetic work, literature research, IR and NMR interpretation.

Conflicts of Interest: The authors declare no conflict of interest.

\section{Abbreviations}

$\begin{array}{ll}5-\mathrm{HT} & \text { serotonin } \\ 5-\mathrm{HT}_{6} \mathrm{R} & \text { serotonin receptor subtype } 6 \\ \text { Anal. } & \text { elemental analysis } \\ \mathrm{IC}_{50} & \text { half maximal inhibitory concentration } \\ \mathrm{IR} & \text { infrared spectroscopy } \\ \mathrm{Ki} & \text { inhibition constant } \\ \mathrm{NMR} & \text { nuclear magnetic resonance spectroscopy } \\ \text { TLC } & \text { thin layer chromatography } \\ \text { TMS } & \text { tetramethylsilane }\end{array}$

\section{References}

1. Kohen, R.; Metcalf, M.A.; Khan, N.; Druck, T.; Huebner, K.; Lachowicz, J.E.; Meltzer, H.Y.; Sibley, D.R.; Roth, B.L.; Hamblin, M.W. Cloning, characterization, and chromosomal localization of a human 5-HT 6 serotonin receptor. J. Neurochem. 1996, 66, 47-56. [CrossRef] [PubMed]

2. Woolley, M.L.; Marsden, C.A.; Fone, K.C. 5-HT 6 receptors. Curr. Drug Targets CNS Neurol. Disord. 2004, 3, 59-79. [CrossRef] [PubMed]

3. Monsma, F.J., Jr.; Shen, Y.; Ward, R.P.; Hamblin, M.W.; Sibley, D.R. Cloning and expression of a novel serotonin receptor with high affinity for tricyclic psychotropic drugs. Mol. Pharmacol. 1993, 43, 320-327. [PubMed] 
4. Heal, D.J.; Smith, S.L.; Fisas, A.; Codony, X.; Buschmann, H. Selective 5-HT 6 receptor ligands: Progress in the development of a novel pharmacological approach to the treatment of obesity and related metabolic disorders. Pharmacol. Ther. 2008, 117, 207-231. [CrossRef] [PubMed]

5. Fisas, A.; Codony, X.; Romero, G.; Dordal, A.; Giraldo, J.; Merce, R.; Holenz, J.; Vrang, N.; Sorensen, R.V.; Heal, D.; et al. Chronic 5- $\mathrm{HT}_{6}$ receptor modulation by e-6837 induces hypophagia and sustained weight loss in diet-induced obese rats. Br. J. Pharmacol. 2006, 148, 973-983. [CrossRef] [PubMed]

6. Upton, N.; Chuang, T.T.; Hunter, A.J.; Virley, D.J. 5-HT 6 receptor antagonists as novel cognitive enhancing agents for alzheimer's disease. Neurotherapeutics 2008, 5, 458-469. [CrossRef] [PubMed]

7. López-Rodríguez, M.L.; Benhamú, B.; de la Fuente, T.; Sanz, A.; Pardo, L.; Campillo, M. A three-dimensional pharmacophore model for 5-hydroxytryptamine6 (5-HT 6 ) receptor antagonists. J. Med. Chem. 2005, 48, 4216-4219. [CrossRef] [PubMed]

8. Vera, G.; Lagos, C.F.; Almendras, S.; Hebel, D.; Flores, F.; Valle-Corvalán, G.; Pessoa-Mahana, C.; Mella-Raipán, J.; Montecinos, R.; Recabarren-Gajardo, G. Extended N-arylsulfonylindoles as 5-HT 6 receptor antagonists: Design, synthesis \& biological evaluation. Molecules 2016, 21, 1070.

9. Mella, J.; Villegas, F.; Morales-Verdejo, C.; Lagos, C.F.; Recabarren-Gajardo, G. Structure-activity relationships studies on weakly basic n-arylsulfonylindoles with an antagonistic profile in the 5- $\mathrm{HT}_{6}$ receptor. J. Mol. Struct. 2017, 1139, 362-370. [CrossRef]

10. Pessoa-Mahana, H.; Recabarren-Gajardo, G.; Temer, J.F.; Zapata-Torres, G.; Pessoa-Mahana, C.D.; Barria, C.S.; Araya-Maturana, R. Synthesis, docking studies and biological evaluation of benzo[b]thiophen-2-yl-3-(4arylpiperazin-1-yl)-propan-1-one derivatives on 5-HT $1 \mathrm{~A}$ serotonin receptors. Molecules 2012, 17, 1388-1407. [CrossRef] [PubMed]

11. You, L.S.; Feng, S.; An, R.; Wang, X.H.; Bai, D.L. Silica gel accelerated aza-michael addition of amines to $\alpha, \beta$-unsaturated amides. Tetrahedron Lett. 2008, 49, 5147-5149. [CrossRef]

12. Hirst, W.D.; Minton, J.A.; Bromidge, S.M.; Moss, S.F.; Latter, A.J.; Riley, G.; Routledge, C.; Middlemiss, D.N.; Price, G.W. Characterization of $\left[{ }^{125} \mathrm{I}\right]-\mathrm{SB}-258585$ binding to human recombinant and native $5-\mathrm{HT}_{6}$ receptors in rat, pig and human brain tissue. Br. J. Pharmacol. 2000, 130, 1597-1605. [CrossRef] [PubMed]

13. Cheng, Y.; Prusoff, W.H. Relationship between the inhibition constant $\left(K_{1}\right)$ and the concentration of inhibitor which causes 50 per cent inhibition $\left(I_{50}\right)$ of an enzymatic reaction. Biochem. Pharmacol. 1973, 22, 3099-3108. [PubMed]

14. Tyson, F.T.; Shaw, J.T. A new approach to 3-indolecarboxaldehyde. J. Am. Chem. Soc. 1952, 74, $2273-2274$. [CrossRef]

15. Aggarwal, R.; Benedetti, F.; Berti, F.; Buchini, S.; Colombatti, A.; Dinon, F.; Galasso, V.; Norbedo, S. A catalytic antibody programmed for torsional activation of amide bond hydrolysis. Chem.-Eur. J. 2003, 9, 3132-3142. [CrossRef] [PubMed]

(C) 2018 by the authors. Licensee MDPI, Basel, Switzerland. This article is an open access article distributed under the terms and conditions of the Creative Commons Attribution (CC BY) license (http://creativecommons.org/licenses/by/4.0/). 